\title{
La réalimentation artificielle des nappes profondes : faisabilité et conséquences
}

\author{
par Michel Detay \\ de la Lyonnaise des Eaux, Cholet \\ et Jean-Luc Bersillon \\ du CIRSEE-ENSG, Nancy
}

\section{LA RÉALIMENTATION ARTIFICIELLE DES NAPPES}

La réalimentation artificielle de nappe (RAN) permet d'équilibrer le bilan des flux et de modifier la qualité de l'eau d'un aquifère en compensant les prélèvements par des apports complémentaires «artificiels ». La réalimentation artificielle peut être considérée comme un fait de l'homme ayant pour objectif d'augmenter l'infiltration naturelle d'eau superficielle vers le réservoir souterrain. La RAN peut se concevoir soit par l'intermédiaire de bassins, de forages, de puits, mais également en modifiant les conditions naturelles d'écoulement d'une rivière ou d'un lac. D'une façon générale la RAN englobe tous les moyens "artificiels » de réalimenta-

(1) Notons que la notion de «nappe profonde » est absente du dictionnaire français d'hydrogéologie. G. Castany et J. Margat, in Editions da BRGM, 249 p. (1977). tion d'une nappe à partir d'eau superficielle. Les techniques de RAN sont utilisées de par le monde depuis plus de deux cents ans. Elles ont de nombreux objectifs que nous avons regroupés en trois classes:

- Gestion active d'un aquifère :

- augmentation de la quantité d'eau dans un aquifère :

- amélioration de la qualité de la ressource aquifère ;

- gestion opérationnelle pour lutter contre des intrusions d'eau salée, des eaux trop minéralisées ou encore de l'eau polluée (barrage hydraulique, confinement); réutilisation d'eaux usées (urbaine ERU ou industrielle ERI), diminution de l'emprise (légale) d'un aquifère exploité à des fins d'eau potable; augmentation du débit des rivières, maintien du débit d'étiage...

- Limitation de la subsidence.

- Gestion des eaux et des réservoirs pétroliers.

Les auteurs introduisent chacune de ces méthodes en illustrant leurs propos par des réalisations significatives de réalimentation artificielle de nappes profondes.

The authors consider as a confined aquifer any non-surface water, that is to say, any aquifer which is not unconfined. Therefore, the depht is not defined as an abstraction related to the distance, but as a structure notion. This concept is indeed more retevant and seems to e nore adapted to the natural environment in applied hydrogeology.

The artificial recharge of confined aquifers is studied through three orientations: active management of the aquifer subsidence control and oiffield exploitation.

Since the technical feasibility of this project proved satisfactory, the relevance of such a process must be considered from an economic standpoint and the costbenefit evaluation remains, in fine, the decisive element. After drawing up the inventory of the sites where artificial recharge of confined aquifers is carried out, the authors propose some refletions on the opportunities brought by this process in order to optimise the water resowice management for the coming centuries. A patrimonial management of the artificial aquifer recharge seems to be the only reasonable solution to supply in water the coming populations. 


\section{LA GESTION ACTIVE DES AQUIFÈRES}

La gestion active des aquifères ou hydrogéologie interventionniste introduit la notion d'action sur les hydrosystèmes [1]. Au-delà du concept, cette volonté d'agir implique, plus que de nouveaux moyens ou de nouvelles méthodes, une philosophie différente, une approche originale, une autre vision de l'hydrogéologie. La gestion active se définit notamment par son spectre de compétence qui ne se limite pas à la géologie ou à l'hydraulique mais englobe la chimie, la physique et surtout la biologie et, enfin, l'informatique en tant qu'instrument de modélisation et de simulation.

La gestion active n'est pas nouvelle, cependant son émergence en tant que telle est récente. Cette prise de conscience s'inscrit dans l'évolution naturelle de la discipline qui est passée progressivement par l'observation, la quantification des flux, la compréhension des phénomènes physico-chimiques et biologiques à l'action sur les hydrosystèmes (cf. fig. l).

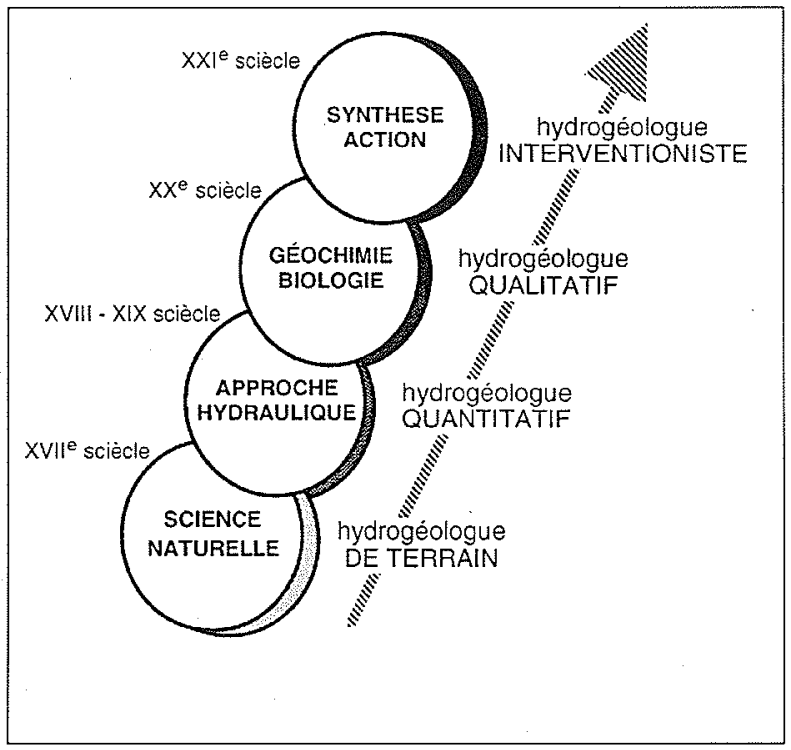

1. Evolution du métier d'hydrogéologue vers la gestion active.

Evolution of hydrogeology over the centuries to groundwater active management.

\subsection{RAN et augmentation du stock}

Un des premiers objectifs de la RAN est d'augmenter le stock d'eau disponible dans un aquifère pour pouvoir le surexploiter de façon temporaire ou permanente. Ce stockage vise généralement à une exploitation différée, la RAN se faisant alors que de l'eau est disponible (période de crue, fonte des neiges), pour être utilisée en période de pointe de consommation.

La figure 2 présente la stratégie d'exploitation de la nappe de Croissy-sur-Seine à l'horizon 2015 compte tenu des besoins identifiés en jour de pointe. Cette stratégie vise à optimiser l'exploitation de la nappe grâce aux installations de réalimentation artificielle de nappe.

L'objectif est de retrouver à l'année $n+1$ un niveau piézométrique équivalent à celui de l'année $n-1$. Ce principe de gestion rationnelle permet d'optimiser l'exploitation de l'aquifère $[2,3]$.

Le Koweît a réalisé un projet de RAN permettant le stockage des excédents saisonniers d'eau issue des usines de dessalement.

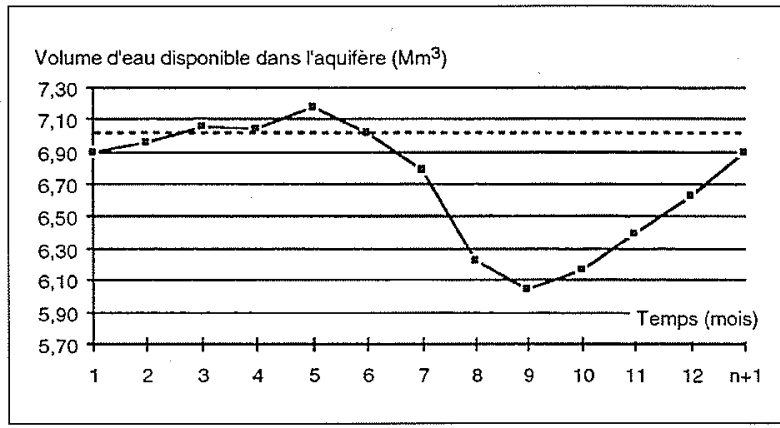

2. Gestion rationnelle de l'aquifère de Croissy-sur-Seine sur une année projet. Simulation des réserves pour l'année 2015. D'après Müller et al., 1993.

Evolution of the aquifer reserve correlated to $A R$ and groundwater withdrawal policies (Croissy-sur-Seine aquifer, 2015 strategy). From Muiller et al., 1993.

Un procédé de gestion active intéressant a été développé dans les karsts. Il consiste à réaliser des barrages dans les cavités karstiques de manière à augmenter la ressource et à pouvoir l'utiliser à des périodes et dans des conditions différentes. Un exemple intéressant a été réalisé en Yougoslavie [4] : le projet Ombla vise à la réalisation d'un barrage karstique d'une soixantaine de mètres de hauteur permettant d'augmenter la capacité de stockage de 25 à 30 millions de mètres cubes $\left(\mathrm{Mm}^{3}\right)$

\subsection{RAN et amélioration dè la qualité}

Pour les distributeurs d'eau et les gestionnaires de l'environnement la réalimentation artificielle de nappe permet non seulement d'augmenter le stock d'eau disponible mais aussi d'en améliorer la qualité. Les phénomènes physico-chimiques et biologiques impliqués ne sont naturellement pas les mêmes selon que l'on s'adresse à des nappes libres, riches en oxygène, ou à des nappes captives, profondes, généralement en anoxie. La qualité du réacteur aquifère sera déterminante en terme d'influence sur les cycles de l'azote et du carbone mais aussi sur les parasites, les virus, les métaux lourds, les pesticides,... $[5,6,7,8]$.

Des études récentes réalisées en France ont montré des abattements de $100 \%$ des parasites entre l'eau brute de RAN (eau de Seine) et l'eau de nappe (cf. tabl. $I$ ).

De nombreuses installations de RAN permettent de réutiliser de l'eau résiduaire urbaine traitée à des fins d'alimentation en eau des populations. Ces installations sont maintenant très nombreuses et sont réalisées avec des effluents secondaires ou tertiaires voire avec de l'eau rendue potable après traitement.

La réutilisation partielle et non contrôlée d'eau usée comme source d'eau brute pour faire de l'eau potable est un phénomène très courant. Le cas le plus fréquent consiste en l'utilisation d'eau de rivière avec la présence de rejets de stations d'épuration à l'amont d'usine de traitement d'eau potable. Se sont les Etats-Unis qui se sont rendus compte le plus tôt de l'importance de ce problème. En 1975 l'EPA considérait déjà que $50 \%$ de l'eau potable provenait d'eau usée recyclée. Compte tenu des besoins toujours croissants les Etats-Unis se sont intéressés de longue date à l'utilisation d'eaux usées pour réalimenter les nappes, lutter contre les invasions salines, contre la subsidence, etc. Du point de vue économique, la solution de l'introduction des eaux usées dans le sol pour lutter contre cette invasion saline est environ trois fois moins onéreuse que l'importation d'eau depuis des contrées éloignées et dix fois moins onéreuse que la dessalination de 1'eau. Les Etats-Unis utilisaient $320 \mathrm{Mm}^{3}$ d'eau usées pour réalimentation de nappe. 
Tableau 1 - Résultats d'une campagne d'analyse parasitologique sur les installations de réalimentation artificielle de nappe de Flins-Aubergenville.

Results from a set of parasitological analyses on the artificial recharge plant of Flins-Aubergenville.

La limite de détection (LD) se situe entre 0,012 et 0,015 kystes par litre.

\begin{tabular}{|l|c|c|c|}
\hline & $\begin{array}{c}\text { Volume } \\
\text { échantillonne } \\
(1)\end{array}$ & $\begin{array}{c}\text { Kyste } \\
\text { Giardia } \\
\left.\text { (par } \mathrm{m}^{3}\right)\end{array}$ & $\begin{array}{c}\text { Kyste } \\
\text { Cryptospori- } \\
\text { dium } \\
\left.\text { (par } \mathrm{m}^{3}\right)\end{array}$ \\
\hline Seine & 400 & 14600 & 5200 \\
Sablière 6 & 659 & 220 & 45 \\
Sablière 8 & 521 & 30 & 60 \\
Forage P1 & 167 & $<$ LD & $<$ LD \\
Forage B5 & 956 & $<$ LD & $<$ LD \\
Forage B1 & 718 & $<$ LD & $<$ LD \\
\hline
\end{tabular}

\subsection{RAN et gestion opérationnelle}

La RAN peut être utilisée à des fins opérationnelles pour lutter contre des intrusions d'eau salée, des eaux trop minéralisées ou encore de l'eau polluée (barrage hydraulique, confinement); réutilisation d'eaux usées (urbaine ERU ou industrielle ERI), diminution de l'emprise (légale) d'un aquifère exploité à des fins d'eau potable; augmentation du débit des rivières, maintien du débit d'étiage... $[9,10,11$, 12].

D'une façon générale la RAN à finalité eau potable est bien connue. Cependant dans la mesure où les distributeurs d'eau utilisent généralement des nappes libres, la RAN ne revêtant pas de finalité eau potable dans des nappes profondes captives est beaucoup moins bien étudiée donc beaucoup moins connue. La finalité de la RAN est donc le phénomène déterminant.

$R A N$ avec de l'eau pluviale : aux Etats-Unis, au Japon, en France, en Belgique, des installations de RAN à partir d'eau pluviale ont été mises en place. En effet, à la suite du développement de l'urbanisation qui entraine une imperméabilisation sans cesse accrue de la surface du sol, les phénomènes de ruissellement peuvent prendre une ampleur considérable. Il devient alors difficile de se débarrasser des eaux de pluie. Par ailleurs, le ruissellement se fait au détriment de l'infiltration et on peut aboutir à une sous-alimentation naturelle de la nappe. La RAN des eaux de ruissellement permet d'atteindre un double objectif : éliminer un volume important d'eau sans avoir à le traiter, favoriser la recharge des nappes.

RAN ERU : de nombreux projets de RAN à partir d'eaux usées se sont développés sur la planète. Généralement ces installations visent à épurer l'eau puis à la réinjecter. Plus l'épuration sera importante meilleure sera la qualité de l'eau dans la nappe.

RAN ERI : depuis 30 ans l'injection dans des nappes profonde est la méthode la plus employée aux Etats-Unis pour se débarrasser des eaux usées industrielles [13]. Approximativement $3,8 \mathrm{Mm}^{3}$ d'effluents industriels sont injectés chaque année dans des nappes profondes entre 400 et $1500 \mathrm{~m}$ [14].

RAN par injection d'eau chaude: bien qu'elles ne soient pas considérées comme des eaux usées ou polluées aux sens bactériologique ou chimique, leur réintroduction dans le milieu naturel reste délicat. En effet, la réintroduction d'eau chaude peut entraîner des effets désastreux sur la faune et la flore aquatique. Ce problème se pose notamment en aval des centrales nucléaires ou d'autres industries utilisant des cirçuits d'eau de refroidissement Une des solutions consiste à réinjecter ces eaux chaudes dans les formations aquifères profondes. Ce procédé a également été utilisé pour du stockage d'énergie thermique.

Comme nous l'avons vu la faisabilité de la gestion active des nappes profondes n'est plus à démontrer. Dans le cadre d'une gestion patrimoniale, c'est-à-dire visant à rendre à nos enfants un milieu naturel dans l'état dans lequel nous aurions aimé le trouver, une typologie liée à la finalité de la RAN semble se dégager. Dans toutes les techniques liées à l'alimentation en eau des populations l'aquifère est contrôlé et ses propriétés sont protégées de manière à ne pas mettre en péril l'outil épuratoire. Dans ce contexte le patrimoine est sauf. A l'opposé les techniques visant à se débarrasser d'un déchet encombrant, généralement d'origine industrielle, risquent de mettre en péril le milieu à court ou moyen terme.

\section{III 圈 CONTRÔLE DE LA SUBSIDENCE}

La subsidence intervient généralement dans des terrains surmontant un aquifère captif profond dont le niveau piézométrique (i.e. la pression) a considérablement baissé à cause de sa surexploitation [15]. Dans la majorité des cas l'aquifère étant captif la RAN par bassin d'infiltration dans un secteur en subsidence n'est pas praticable sauf cas particuliers où la zone d'alimentation de la nappe est relativement proche. La RAN n'est utilisable que par forage de réinjection pour remettre en pression l'aquifère captif. Bien que cette technique soit très onéreuse elle peut être la seule capable de résoudre le phénomène de subsidence.

La subsidence de la ville de Shanghai (Chine) a été signalée dès 1921. La ville se trouve sur une série sédimentaire de $300 \mathrm{~m}$ d'épaisseur renfermant une nappe captive exploitée. La période de subsidence maximum ( $98 \mathrm{~mm} / \mathrm{an})$ concerne les années 1956-1959. En 1965, la ville avait baissé dans certains secteurs de $2,63 \mathrm{~m}$; les surfaces concernées sont considérables avec $121 \mathrm{~km}^{2}$ dans lesquels la subsidence était de plus de $0.5 \mathrm{~m}$. En 1965 la subsidence était de $23 \mathrm{~mm} / \mathrm{an}$. En 1964, la décision a été prise de réinjecter de l'eau de rivière dans le principal aquifère. Cette action a été relayée dès 1966 par une centaine d'industries qui ont participé à l'effort de réalimentation en rechargeant l'aquifère captif par l'intermédiaire de forages de réinjection. L'arrêt de la subsidence a été quasiment immédiat, dès 1966 la ville remontait de $6,3 \mathrm{~mm} / \mathrm{an}$; en 1976 la ville était remontée de $34 \mathrm{~mm}$.

Il convient de citer le projet de lutte contre la subsidence de Venise (Italie) où certains experts avaient suggéré de repressuriser la nappe profonde. Venise est bâtie sur une série sédimentaire quaternaire de plus de $1000 \mathrm{~m}$ d'épaisseur où alternent des horizons aquifères captifs et des aquitards. La subsidence a notamment été provoquée par l'exploitation de quatre horizons aquifères parmi six présents dans la formation. L'étude historique de l'exploitation des aquifères profonds met en évidence plusieurs périodes pendant lesquelles il est possible de corréler surexploitation et subsidence (cf. fig. 3).

Au Japon, on recense une dizaine de zones sujettes à la subsidence par surexploitation d'aquiferes profonds. Sept villes ont été amenées à renoncer à l'exploitation de leurs ressources souterraines et à traiter de l'eau superficielle pour arrêter la subsidence. Dans de nombreux cas, de l'eau usée a été traitée et réinjectée. A Niigata une expérience intéressante de réalimentation d'un aquifère profond riche en méthane a été tentée de 1960 à 1963 et l'expérience a été jugée satisfaisante [16].

Aux Etats-Unis on note classiquement 18 sites sujets à une subsidence importante. Dans six d'entre eux la solution a été d'abandonner l'exploitation des aquiferes profonds et utiliser de l'eau superficielle pour l'alimentation en eau potable. Ces mesures ont permis une relative stabilisation de la pression artésienne et une limitation de la subsidence (Vallée de Santa Clara et Vallée de San Joaquin en Califor- 


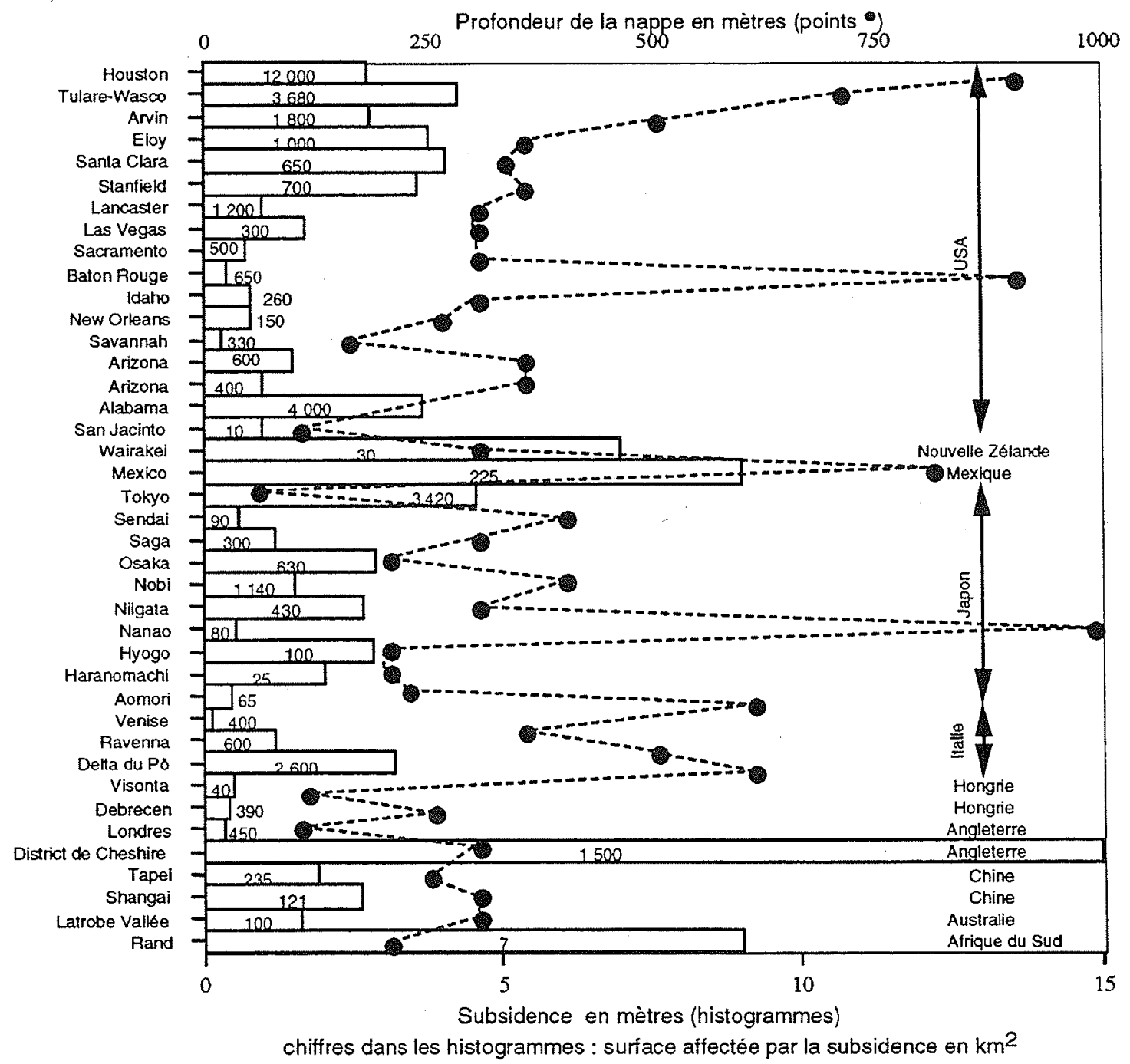

3. Bilan de la subsidence dans le monde. Subsidence in the world: state of the art.

nie ; vallée de Las Vegas au Nevada ; secteur de HoustonGalveston au Texas). La repressurisation des aquifères n'a été nécessaire que dans le cas de la vallée de Santa-Clara.

L'analyse de la figure 3 montre l'importance des phénomènes de subsidence tant sur le plan de l'altimétrie que sur celui des superficies concernées. La RAN des nappes profondes (la moyenne se situant à 380 mètres de moyenne) apparaît être un moyen efficace de lutte contre la subsidence, cependant les caractéristiques mécaniques des roches peuvent être modifiées lors de la subsidence et il est généralement impossible de retrouver les caractéristiques initiales.

\section{RAN DANS L'EXPLOITATION PÉ- TROLIÈRE ET GAZIÈRE}

L'eau en exploitation pétrolière est soit un outil de production comme cela a été le cas jusqu'au début des années 80 , soit un outil pour limiter voire arrêter les phénomènes de subsidence constatés dans un certain nombre d'exploitations, soit encore un déchet de la production.

En temps qu'outil de production, l'eau a été utilisée afin d'assurer la récuperation tertiaire de pétrole. Cette pratique a été abandonnée lorsque le prix du baril de pétrole est redescendu en dessous de 18 US $\$$.

La technique utilisée consistait en l'accomplissement des phases suivantes:

- le «décrochage » de l'huile de la surface de la roche au moyen de tensioactifs,

- la formation d'un «bouchon» de polymères,

- la mise en pression par injection de fluide dont l'eau pour pousser ce bouchon.

A ce dernier titre, les techniques utilisées et les caractéristiques de l'eau relèvent tout à fait de la RAN.

Comme dans le cas de la surexploitation des nappes profondes, l'abstraction de pétrole ou de gaz des couches productrices peut aussi provoquer un affaissement des couches supérieures. 
Le champ pétrolier de Wilmington (Californie du Sud) est un exemple classique de contrôle de la subsidence par des forages de réinjection. La repressurisation de la zone de production pétrolière pour augmenter la production d'huile et pour lutter contre la subsidence est devenue très tôt (1958) une technique classique. En 1969, $175000 \mathrm{~m}^{3}$ $\left(1,1.10^{6}\right.$ barils) d'eau étaient injectés chaque jour dans la zone de production de pétrole. La zone de subsidence a été réduite de 58 à $8 \mathrm{~km}^{2}$. Localement la surface du sol est remontée de $30 \mathrm{~cm}$. En $1975,80 \mathrm{Mm}^{3}$ avaient été injectés dans l'aquifère pour contrôler la subsidence et produire du pétrole [17]. Ce programme a permis de réduire la vitesse de subsidence de $75 \mathrm{~cm} / \mathrm{an}$ en 1958 à une vitesse nulle en 1968 .

Il existe d'autres cas de subsidence associée à l'exploitation pétrolière et gazière. L'un des plus surveillés est sans doute le cas du gisement de gaz de Gröningen en Hollande [18]. Ce cas est particulièrement intéressant du fait de sa localisation. Il est encore aujourd'hui sous étroite surveillance [19]. Le plus récent est sans doute celui du champs pétrolifère d'Ekofisk en Norvège. La subsidence associée à l'exploitation de ce champ rend l'exploitation plus difficile et plusieurs solutions dont la réinjection d'eau ont été envisagées [20].

La production de pétrole est souvent associée à la production d'eau dont il faut se débarrasser. C'est le cas des champs pétrolifères de la Mer du Nord où plusieurs centaines de milliers de barils d'eau sont produits chaque jour. Les contraintes environnementales demandent un traitement qui peut être coûteux. Jusqu'à récemment, la réinjection de ces eaux était subordonnée à un traitement pour l'élimination des particules et des gouttelettes d'huile. Cette méthode d'élimination des eaux de production pétrolière gagne cependant en popularité [21]. En effet, le coût de cette méthode n'est guère plus élevé que les autres méthodes d'élimination. De plus, il semble que la qualité de l'eau à réinjecter ne soit pas si cruciale. Plusieurs cas de maintien du débit de réinjection ont été constatés lors de baisse de performance des unités de traitement. Ces données suggéreraient que le modèle d'écoulement radial du fluide lors de la réinjection doit être remis en cause.

\section{$\mathrm{V}$ - CONCLUSION}

La RAN des nappes profondes est gouvernée par trois compétences bien différentes faisant appel à deux phénomènes physiques distincts : le transfert de masse et le transfert de pression. Actuellement le transfert de masse est essentiel dans le cadre de réutilisation de l'eau (RAN à finalité eau potable, réutilisation d'eaux usées....); pour cet objectif les phénomènes physico-chimiques et biologiques, des différents réacteurs et interfaces, sont sollicités pour épurer l'eau. A l'opposé, dans le transfert de pression l'eau est employée en tant que fluide dont la caractéristique essentielle devient l'incompressibilité : lutte contre la subsidence. Enfin, masse et pression sont utilisées dans l'exploitation pétrolière où l'eau intervient en tant que fluide de substitution pour permettre la récupération du pétrole.

La RAN s'avère être une technique très performante de gestion active d'un réservoir souterrain. Elle devrait être appelée à se développer dans le futur notamment dans le domaine de la réutilisation des eaux usées. Le stockage souterrain en nappe présente de nombreux avantages pour répondre aux besoins en eau à court et moyen terme. Comme nous l'avons vu le bilan coût-avantage est le principal élément décisionnaire. Cependant ce bilan évolue dans le temps et rien n'interdit de croire que demain la RAN ne sera pas utilisée pour diluer des nappes profondes actuellement salées, impropres à la consommation, pour les réutiliser à des fins d'eau potable. Dans tous les cas la RAN doit être utilisée de manière consciente dans une perspective de mâ̂trise collective du patrimoine afin de ne pas gravement compromettre le potentiel aquatique des générations futures. Il est de notre responsabilité que les aménageurs et les décideurs intègrent ces enjeux.

\section{Bibliographie}

[1] Detay M., COLlin J.-C. (1995). - Introduction à la gestion active des aquiferes : concept et philosophie (Editorial du colloque AIH 1995), in Hydrogéologie, 1, 3-11.

[2] DETAY M. (1995). - Le rôle de la réalimentation artificielle de nappe dans la gestion active des aquiferes, in Hydrogéologie, $1,57-65$

[3] Múller O., Detay M. (1993). - Modélisation du comportement des éléments azotés en aquifere alluvial influencé : importance de l'interface surface-nappe, in Hydrogéologie, 1, 3-19.

[4] Milanovic P.T. (1988). - Artificial underground reservoirs in the karst experimental and project exemple, in proc. $21 \mathrm{st}$ IAH Congress, 1, 76-87.

[5] Detay M., Dumoutier N., Haeffner H., Vignier V. (1994). Incidence de la réalimentation artificielle de nappe sur la qualité de la ressource, in HYDROTOP 94, 1, 357-366.

[6] DETAY M. (1994). - Rational ground water reservoir management: the role of Artificial Recharge, in proceedings of Second International Symposium of arificial recharge of groundwate; Orlando, Floride, USA 231-240.

[7] DEtay M. (1993). - Le forage d'eau, réalisation, entretien, réhabilitation. Masson Ed.

[8] Detay M., Habfaner H., Bersillon J.-L. (1995), - The role of artificial recharge in groundwater active management quality, aquifer storage and recovery, environmental effects, and economical perspectives, in proceedings of the $7 \mathrm{~h} \mathrm{Sym}$ posium on Artificial Recharge of Groundwate; Temple, Ari zona, USA 83-97.

[9] Bersillon J.-L., Detay M.. Feuardent J.-P., Vignier V (1994). - Adsorption-désorption de l'atrazine en zone saturée. Application aux roches aquifères de la région parisienne : craie sénonienne et calcaire de Champigny, in Comptes Rendus de l'Académie des Sciences, Paris, t. 318. Sérje II, 1357-1363.

[10] Chambolle T., Deray M., Suzanne P. (1994). - Regard rétrospectif et prospectif sur l'hydrogéologie à l'aube de troisième millénaire, in Hydrogéologie, 1, 19-23.

[11] Suzanne P., Detay M. (1993). - Méthodes spécifiques appliquées à la protection des eaux souterraines en Ile-deFrance, in proc. Gestion des ressources et protection de l'eau, CEFICI, 20-21 octobre, JE 393, 10 p.

112) DETAY M. (1991). - Techniques modernes d'action sur les eaux souterraines, bilan et perspectives, in Rapport Général de la Question II des XXI' Journées de I'Hydraulique, Colloque de la Société Hydrotechnique de France, G.G.II, 1-13.

[13] Nativ R., Hemo I., Wenberger G. (1994). - Injection of industrial wastewater in Israel : siting criteria for deep injection wells and associated problems, in Joumal of Hydrology; $163,299-323$

[14] Gordon W., Bloom J. (1986). - Deeper problems - limits to underground injection as a hazardous waste disposal method, in actes du congrès "subsurface injection of liquid waste », 35 mars 1986, New Orleans, NWWA, Dublin, OH, 3-39.

[15] POLAND J. F. (1984). - Guidebook to studies of land subsidence due to groundwater withdrawal, in Studies and reports in hydrology; 40, $305 \mathrm{p}$.

[16] IsHWhad, Yasufumi (1969). - Experiments on water injection in the Nigata gas field, in IAHS-AISH Pub 89, 629-634

[17] Gates G. L., Caraway W.H., Lechtenberg H.J. (1977). Problems in injection of waters in Wilmington oil field, California, in IAHS-AISH Pub. 121, 319-324.

[18] Schoonbeek J.B. (1976). - Land subsidence as a result of natural gas extraction in the Province of Groningen, in $S P E$ 5751 .

[19] Mobach E., Gussinklo H.J. (1994). - In simu reservoir compaction monitoring in the Groningen Field. EUROCK 94. Rock Mechanics in Petroleum Engineering Conference, Delft, Holland, 29-31 Aug. 1994, in Proceedings, 535-547. Balkema edit, Rotterdam.

[20] Wiborg R., JEwhurst J. (1986). - Ekofisk Subsidence Detailed and Solutions Assessed, in Oil \& Gas Joumal.

21] Palge R.W., Murray L.R. (1994). - Reinjection of Produced Water. Field Experience and Current Understanding. EUROCK 94. Rock Mechanics in Petroleum Engineering Conterence, Delft, Holland, 29-31 Aug. 1994, in Proceedings, 731-738. Balkema edit., Rotterdam. 